2. Pre-operative PET scans play an undoubted role in selecting patients for surgery but thus far in our service, they don't appear to be associated with significantly less RD because of the problem of microscopic metastases.

Abstract P195 Table 1 Temporal trends and distribution of recurrent disease following lung cancer surgery

\begin{tabular}{lcll}
\hline Site & $\mathbf{N}=\mathbf{5 8}$ & Range (months) & Mean \\
\hline Lung & 24 & $1-44$ & 19.8 \\
Brain & 10 & $2-23$ & 12.3 \\
Bone & 8 & $1-44$ & 23.0 \\
Liver & 2 & $14-34$ & 24.0 \\
Mediastinum & 3 & $6-44$ & 29.7 \\
Lymph nodes & 4 & $12-18$ & 14.8 \\
Skin & 2 & $3-32$ & 16.5 \\
\hline
\end{tabular}

\section{P196 CRYO-RECANALISATION VIA DAY-CASE FLEXIBLE BRONCHOSCOPY FOR CENTRAL AIRWAY OBSTRUCTION}

doi:10.1136/thx.2010.151043.47

B Ng, U Oltmanns, C Hardy, C Yip, M Slade. Papworth Hospital, Cambridge, UK

Introduction and Objectives The use of a flexible cryoprobe to effect bronchial recanalisation inpatients with central airway obstruction (CAO) has previously been described, in patients under general anaesthesia undergoing rigid bronchoscopy (C Schumann et al. J Thorac Cardiovasc Surg 2010;139:997-1000). Few data on flexible bronchoscopic cryorecanalization have been published.

Methods All flexible interventional bronchoscopic procedures were recorded prospectively in adatabase, and data extracted on all procedures in which cryo-recanalisation (Erbokryo, ERBE UK Ltd) was attempted. Data collected included demographics, diagnosis, indication for procedure and site of tracheobronchial abnormality, techniques used, complications and the following data pre- and post-procedure: performance status, $\mathrm{FEV}_{1}$, patient location. The procedure duration, defined as the time in minutes between initial bronchoscope insertion and final bronchoscope withdrawal, was recorded in 23 cases. Bronchoscopies were performed in Oxford and Papworth by, or under the direct supervision of, a single operator (MS).

Results Between May 2006 and July 2010, 54 procedures were performed in 46 patients ( 13 female, 33 male). The median age was 69 (range 24-94). The underlying diagnosis was lung cancer in 39 procedures, endobronchial metastases in 13 and benign disease in 2 (post tracheostomy granulation tissue in 1, thrombus in 1). All patients had CAO affecting a lobar or more proximal bronchus. There was a mean improvement in FEV1 of 0.28L (mean (SD) FEV 1 pre-procedure $1.56 \mathrm{~L}(0.71 \mathrm{~L})$, mean $(\mathrm{SD}) \mathrm{FEV} 1$ post procedure $1.84 \mathrm{~L}$ (0.80), $\mathrm{p}=0.001$, paired t-test). Median performance status was 1 both before and after the procedure. In $49 / 54$ procedures (91\%) the patients were treated as day-cases, and $5 / 54(9 \%)$ as inpatients. Bleeding complications occurred in two patients. In both cases bleeding of $>50 \mathrm{mls}$ occurred, which was controlled using epinephrine via the bronchoscope. The median procedure duration was 20 (range 7-58) min.

Conclusions Day-case cryorecanalization via flexible bronchoscopy appears to offer effective improvement in lung function in patients with $\mathrm{CAO}$ owing to endobronchial lesions. Procedure duration is short, and complication rates appear acceptable. We believe that cryo-recanalisation using flexible cryoprobes deserves more widespread use in centres specialising in interventional bronchoscopy.

\section{Clinical studies in obstructive sleep apnoea P197 THE EFFECT OF CPAP THERAPY ON INSULIN SENSITIVITY AND CV RISK FACTORS IN PATIENTS WITH OSA}

doi:10.1136/thx.2010.151043.48

M Paracha, D Ahearn, W Flight, V Gupta, M Abdelhalim. Blackpool Victoria Hospital, Blackpool, UK

In a prospective study, we assessed the effect of CPAP therapy on the insulin sensitivity and CV risk factors such as blood pressure (BP), glycaemia status, and lipid profile in patients with obstructive sleep apnoea (OSA).

Only patients with confirmed presence of cardinal features and diagnostic apnoea/hypopnoea index (AHI) for OSA were enrolled in the study. All patients were studied before the initiation of CPAP therapy. Following an initial screening visit before CPAP treatment, patients were then studied at 6 and 12 weeks following the initiation of CPAP.

We studied 41 patients with confirmed OSA, aged $56 \pm 13$ (mean \pm $\mathrm{SD}$ ) years, 33 male and 8 female, who had their weight, BP and fasting blood samples checked in a screening visit and two study visits, at least 6 weeks apart. The fasting blood samples were used to assess fasting plasma glucose, $\mathrm{HbA}_{1 \mathrm{c}}$, fasting insulin, lipid profile, and CRP.

Following CPAP treatment baseline systolic BP had significantly improved compared to BP following CPAP therapy (135 \pm 17 vs $123 \pm$ $12 \mathrm{~mm} \mathrm{Hg}, \mathrm{p}=0.001)$ and so did diastolic BP (83 \pm 9 vs $77 \pm 9 \mathrm{~mm}$ $\mathrm{Hg}, \mathrm{p}=0.01)$. There were also improvement in fasting plasma glucose $(5.6 \pm 1.7$ vs $4.9 \pm 2.0 \mathrm{mmol} / \mathrm{l}, \mathrm{p}>0.05)$ and $\mathrm{HbA}_{1 \mathrm{c}}(5.8 \pm 1.3$ vs $5.4 \pm$ $1.7 \%, \mathrm{p}>0.05)$, but that was not statistically significant. There had been non-significant improvement of fasting insulin level $(17.9 \pm 25$ vs $12.7 \pm 15.5 \mathrm{pmol} / \mathrm{l}, \mathrm{p}>0.05)$, but no improvement on insulin sensitivity. Lipid profile had also improved, as total cholesterol was decreased ( $5.2 \pm 2.0$ vs $4.8 \pm 1.9 \mathrm{mmol} / \mathrm{l}, \mathrm{p}>0.05)$, and so did triglycerides $(2.7 \pm 4.4$ vs $2.0 \pm 2.0 \mathrm{mmol} / \mathrm{l}, \mathrm{p}>0.05)$, but these changes were not statistically significant. CRP had also improved but that was not statistically significant ( $4.5 \pm 10$ vs $2.4 \pm 2.0 \mathrm{mg} / \mathrm{l}, \mathrm{p}>0.05)$.

In conclusion, this study demonstrates that CPAP therapy in patients with OSA can result in significant improvement in systolic and diastolic BP, as well as non-significant improvement in lipid profile and glycaemia status.

\section{P198 EFFECTS OF OXYGEN THERAPY ON CENTRAL SLEEP- DISORDERED BREATHING IN INFANTS WITH PRADER-WILLI SYNDROME}

doi:10.1136/thx.2010.151043.49

${ }^{1} \mathrm{D}$ S Urquhart, ${ }^{2} \mathrm{P}$ Wales, ${ }^{2} \mathrm{C}$ Parsley, ${ }^{2} \mathrm{~S}$ Suresh. ${ }^{1}$ Royal Hospital for Sick Children, Edinburgh, UK; ${ }^{2}$ Mater Children's Hospital, Brisbane, Australia

Background Children with Prader-Willi syndrome (PWS) are known to have sleep-disordered breathing. In addition to hypersomnolence and obstructive sleep apnoea, central respiratory control abnormalities may be present from infancy. The aims of this study were to describe breathing patterns in infants with PWS, and the effects of supplemental oxygen in this group.

Methods Children with PWS attending a tertiary sleep clinic underwent full polysomnographic studies either to investigate persisting neonatal oxygen requirement, or as screening for sleepdisordered breathing. Continuous oxygen saturations $\left(\mathrm{SpO}_{2}\right)$ and transcutaneous carbon dioxide $\left(\mathrm{tcCO}_{2}\right)$ were recorded. Central and obstructive events were defined in accordance with the American Academy of Sleep Medicine (AASM) 2007 scoring rules. Children who had significant hypoxia associated with central events were started on supplemental oxygen during sleep and followed at 3monthly intervals with split-night studies (periods in both air and supplemental oxygen). Paired $t$-tests were used to compare sleep data in air and oxygen arms for our subject cohort. 
Results 30 split-night studies were undertaken on 10 infants (8 female) aged 0.06-1.79 (mean 0.79, SD 0.44) years. At baseline (ie, air), children with PWS had a mean (SD) central apnoea index (CAI) of 6.9 (6.3) per hour, with accompanying falls in $\mathrm{SpO}_{2}$. Oxygen therapy led to statistically significant reductions in CAI, as well as improved $\mathrm{SpO}_{2}$ (Abstract P198 Table 1). No significant change in the number of obstructive events was noted.

Abstract P198 Table 1 Effect of oxygen therapy on sleep-disordered breathing in infants with PWS

\begin{tabular}{|c|c|c|c|}
\hline & Air & Oxygen & p-Value* \\
\hline$\%$ Study with $\mathrm{SpO}_{2}<90 \%$ & $1.5(3.8)$ & $0.2(0.4)$ & $<0.05$ \\
\hline Lowest $\mathrm{SpO}_{2}(\%)$ & $77(8)$ & $89(6)$ & 0.06 \\
\hline Longest central apnoea (s) & $10.3(4.2)$ & $9.8(3.7)$ & 0.68 \\
\hline Maximum $\mathrm{tcCO}_{2}(\mathrm{~mm} \mathrm{Hg})$ & $56.5(7.5)$ & $55.3(12.5)$ & 0.58 \\
\hline Central apnoea index (CAI) & $6.9(6.3)$ & $3.7(4.2)$ & $<0.001$ \\
\hline Obstructive event index (OEI) & $3.9(4.1)$ & $5.4(8.7)$ & 0.37 \\
\hline
\end{tabular}

*Paired $t$-test.

Discussion Infants with PWS have sleep-disordered breathing problems, which are predominantly central in origin, and cause significant hypoxia in some patients. Improvements in both central event indices and oxygenation were noted on oxygen therapy. Longitudinal work with this patient group would help to establish timing of onset of obstructive symptoms. Whether early recognition of central hypoventilation, and correction with oxygen alter the evolution of respiratory dysfunction and excessive daytime somnolence in later life remains to be seen.

\section{P199 DIABETIC MACULAR OEDEMA (DME) AND OBSTRUCTIVE SLEEP APNOEA (OSA) - VISUAL IMPROVEMENT POST CPAP THERAPY. PROOF OF PRINCIPLE STUDY}

doi:10.1136/thx.2010.151043.50

${ }^{1} \mathrm{R}$ H Mason, ${ }^{2} \mathrm{C}$ A Kiire, ${ }^{2} \mathrm{~A}$ Bolton, ${ }^{2} \mathrm{~L}$ Smith, ${ }^{2} \mathrm{~V}$ Chong, ${ }^{1} \mathrm{~J}$ R Stradling. ${ }^{1}$ Oxford Sleep Unit, The Churchill Hospital, Oxford, UK; ${ }^{2}$ The Eye Hospital, Oxford, UK

Introduction and Objectives DME is an important cause of visual loss and is more advanced in patients with coexistent OSA. ${ }^{1}$ OSA is very common in individuals with $\mathrm{DME}(54 \%)$, compared to unselected patients with type II diabetes $(23 \%)^{2}$ and age matched controls $(8 \%)^{3}$. This study was designed to see if CPAP could improve vision in patients with DME and coexistent OSA, perhaps via a reduction in intermittent hypoxia and/or blood pressure oscillations.

Methods 35 patients with DME (identified by ocular coherence tomography, OCT) and OSA (oxygen desaturation index $>10$, or apnoea hypopnoea index $>15$ ) were identified. Visual acuity (VA, $\log$ MAR, similar to the Snellen chart) and OCT measurements were made twice at baseline (pre-CPAP), 3 and 6 months (post-CPAP).

Results 32 patients (17 males) participated; 4 withdrew. 28 have 3month follow-up data and, 24 have 6 -month data. Average (SD) age 66.6 years, (8.3), BMI $31.8 \mathrm{Kg} / \mathrm{m}^{2}$ (6.7), HbA1c $7.5 \%$ (1.4\%), ESS 7.9 (4.6), ODI 20.9 (14.8) and AHI 19.0(14.5). CPAP compliance was averaged over the 6 months and a median split into 'high' and 'low' compliers performed ( $>$ and $<2.5 \mathrm{~h} / \mathrm{n}$ ). At 3 months VA improved significantly in both high $(p=0.009)$ and low compliers $(p=0.001)$. This was only sustained at 6 months in high compliers, $p=0.004$. (Low compliers $p=0.52$ ). There was no significant reduction in macular oedema at either 3 or $n$.

Conclusions This hypothesis-generating uncontrolled study indicates that continued use of CPAP in individuals with DME and OSA was associated with sustained improvement in visual acuity. This result provides justification to perform an RCT and suggests that
logMAR should be the primary endpoint whereas OCT measurements appear uninformative.

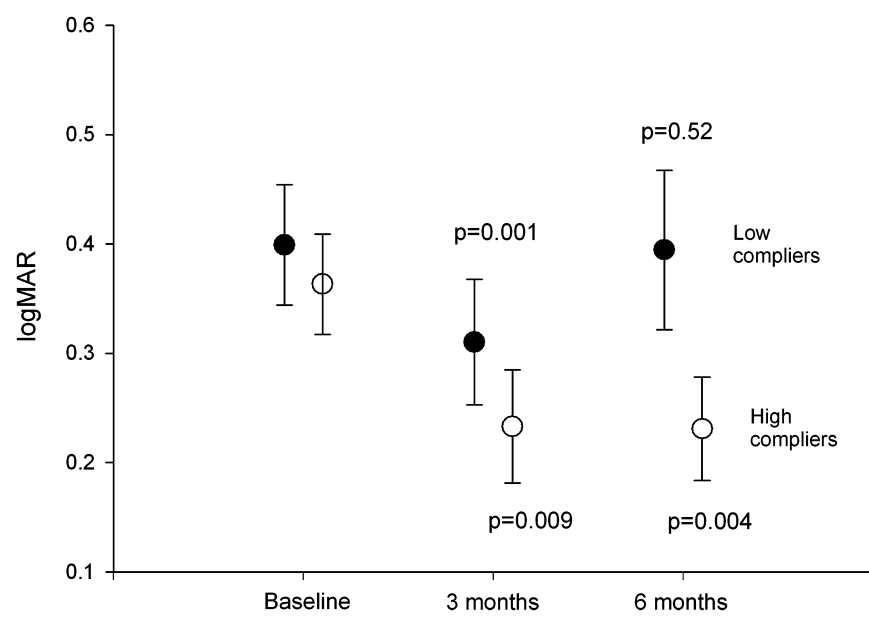

Abstract P199 Figure 1 LogMAR after CPAP use, high versus low compliers (mean \pm SEM).

\section{REFERENCES}

1. West SD, et al. The prevalence of retinopathy in men with type 2 diabetes and obstructive sleep apnoea. Diabetic Medicine 2010;27:423-30.

2. West SD, et al. Prevalence of obstructive sleep apnoea in men with type two diabetes. Thorax 2006;61:945-50.

3. Stradling JR, Crosby JH. Predictors and prevalence of obstructive sleep apnoea and snoring in 1001 middle aged men. Thorax 1991:46:85-90.

SNORING AND CAROTID ATHEROMA: NO ASSOCIATION

doi:10.1136/thx.2010.151068.1

${ }^{1} \mathrm{R}$ H Mason, ${ }^{2} \mathrm{Z}$ Mehta, ${ }^{2} \mathrm{P}$ M Rothwell, ${ }^{1} \mathrm{~J}$ R Stradling. 'Oxford Sleep Unit, The Churchill Hospital, Oxford, UK; ${ }^{2}$ Department of Clinical Neurology, John Radcliffe Hospital, Oxford, UK

Background Obstructive Sleep apnoea (OSA) has increasingly been linked to cardiovascular endpoints including hypertension, stroke, myocardial infarction, and carotid atherosclerosis. Snoring is strongly associated with OSA. More recently, the snoring component of OSA, by producing local arterial trauma, has been independently linked to the presence of carotid but not femoral atheroma, ${ }^{1}$ and to acute carotid trauma in an animal model. ${ }^{2}$ The aim of this study was to identify whether this relationship could be established retrospectively in a high vascular risk cohort of individuals with a recent cerebrovascular event (TIA or stroke).

Method Participants with a recent TIA or stroke in the Oxford Vascular Study (OXVASC) completed an entry questionnaire which included frequency of snoring (never, rarely (1-2/year), occasionally (4-8/year), sometimes ( $1-2 /$ month), often ( $1-2 /$ week), usually (3-5/week) and always (every night). A subset of 316 individuals (aged 62-84years), with both a completed snoring questionnaire and carotid Doppler data, were included. This information was used to retrospectively assess a potential relationship between presence and severity of snoring, and degree of carotid artery stenosis and plaque type (echogenic/echolucent/mixed, calcified/non-calcified and smooth/irregular).

Results 160 out of 316 participants were male, mean (SD) age 73.2 (11.22). No significant association could be identified between intensity of self-reported snoring and degree of carotid stenosis or plaque morphology.

Conclusions No significant association could be identified between snoring and carotid atheroma. There are no obvious explanations for the discrepancy between this study and previous work, except that the original study measured snoring objectively during a one night 